\title{
Phase Conjugation Using Four-Wave Mixing in Fast Green FCF Dye-Doped Gelatin Film
}

\author{
M. H. Majles Ara, ${ }^{1}$ S. Mehrabani, ${ }^{2}$ and R. Malekfar ${ }^{2}$ \\ ${ }^{1}$ Photonics Laboratory, Physics Department, Tarbiat Moallem University, 49 Moffateh Ave, Tehran, Iran \\ ${ }^{2}$ Physics Department, Faculty of Science, Tarbiat Modares University, 49 Moffateh Ave, Tehran, Iran
}

Correspondence should be addressed to M. H. Majles Ara, majlesara@gmail.com

Received 30 May 2009; Revised 6 November 2009; Accepted 7 December 2009

Recommended by Majid Ebrahim-Zadeh

We present optical phase conjugation based on the degenerate four-wave mixing (DFWM) arrangement in gelatin films doped with acid blue 3 dye (FAST GREEN FCF) using He-Ne laser of total power $50 \mathrm{~mW}$. Various parameters which influence the phaseconjugate (PC) signal during the DFWM process were studied (angle of between the forward pump and the probe beam, transition of gelatin film, reflectivity as a function of forward and backward beam). A maximum phase-conjugate beam reflectivity of about $0.02 \%$ has been observed in these dye-doped gelatin films. PACS: 42.65.-k, 42.65.Hw.

Copyright (c) 2009 M. H. Majles Ara et al. This is an open access article distributed under the Creative Commons Attribution License, which permits unrestricted use, distribution, and reproduction in any medium, provided the original work is properly cited.

\section{Introduction}

Nonlinear optical phase conjugation by degenerate fourwave mixing (DFWM) is an important technique with applications in many fields of science and technology [1]. When two counter propagating and intense light beams interact with a nonlinear medium, together with a less intense third one, a fourth beam is generated from the medium, which will be the phase conjugation of the third beam. This technique is called four-wave mixing. The main applications of degenerate DFWM techniques are nonlinear spectroscopy, real time holography, and phase conjugation. Phase conjugation by (DFWM) has been demonstrated in numerous nonlinear media $[2,3]$. The organic molecules exhibit large polarizabilities because exited $\pi$-bond electrons are delocalized and hence easily polarizable [4]. Saturable absorption plays a very important role when dyes are used for the production of phase conjugation light, because $\chi^{(3)}$ is inversely proportional to the saturation intensity [5]. These systems exhibit large third-order susceptibilities. Figure 1 shows the energy level of dyes. These materials can be explained by three-level system [6]. When light is injected, electrons excite from the $S_{0}$ level to the $S_{1}$ level. The dye molecules will switch over to the triplet state. The number of electrons at the $S_{0}$ level is reduced only by the number of electrons occupying the $T_{1}$ level. Therefore, the number of excitable electrons at the $S_{0}$ level is dramatically reduced if the intensity of the injected waves is high at a frequency corresponding to the $S_{0}$ and $S_{1}$ levels energy; the rate of absorption is thus reduced [1]. Thus, absorption becomes a function of intensity. The gratings produced by interference between the probe and pump wave that the period can be determined using the following relation:

$$
\Lambda=\frac{2 \pi}{\kappa_{G}}=\frac{\lambda}{2 n \sin (\theta / 2)},
$$

where $\lambda$ is the laser wavelength. At small $\theta$ angel, the spacing is wider for a transmission grating than for a reflection grating $[7,8]$. The theory of DFWM was formulated for a two-level saturable absorber by Abrams and Lind [9] and was modified to describe a three-level absorber by Silberberg and Bar-Joseph [10]. The phase conjugated reflectivity is then given by

$$
R=\frac{|k|^{2}}{|\alpha+w \cot (w L)|^{2}}
$$


where $w=\left(|k|^{2}-\alpha^{2}\right)^{1 / 2}, \alpha$ is saturated absorption coefficient given by

$$
\alpha=\alpha_{0} \frac{1+I_{1}+I_{2}}{\left[1+2\left(I_{1}+I_{2}\right)+\left(I_{1}-I_{2}\right)^{2}\right]^{3 / 2}},
$$

and $k$ is coupling coefficient given by

$$
k^{*}=2 \alpha_{0} i \frac{\left(I_{1} I_{2}\right)}{\left[1+2\left(I_{1}+I_{2}\right)+\left(I_{1}-I_{2}\right)^{2}\right]^{3 / 2}} .
$$

$I_{1}$ and $I_{2}$ are two pump beam intensities normalized by the saturation intensity $I_{s}[10]$.

\section{Thermal Grating}

Thermal processes can lead to large nonlinear optical effects. Consequently, the illuminated portion temperature of the material increases; the refractive index invariably decreases with increasing temperature [11]. Under steady-state conditions, the heat transport equation reduces to

$$
\frac{\Delta(n L)}{n L}=\frac{1}{n} \frac{\partial n}{\partial T} \Delta T+\frac{1}{L} \frac{\partial L}{\partial T} \Delta T
$$

where $n$ is refractive index and $L$ is sample thickness $[8,12]$.

\section{Experimental Configuration}

Figure 2 shows the standard experimental degenerate fourwave mixing set-up. A $50 \mathrm{~mW}$ He-Ne laser at $632 \mathrm{~nm}$ is used to generate PC from Fast Green FCF. The output beam first is divided into two beams by beam splitter SB1 (10:90) [13]. The transmitted beam split with another beam-splitter BS2 $(50: 50)$ to provide the pump beams which are called $E_{1}, E_{2}$. The beam reflected from BS1 splits by BS3 (50:50) to provide the probe beam $E_{3}$. The Fast Green FCF dye film is exposed simultaneously to all the three beams. The path lengths of all waves reaching the sample are matched well with the coherence length of the laser [3], so that they are coherent at the sample. PC travels opposite to the direction of the probe beam. The intensity of phase conjugation wave is measured by a photodetector. The ratio of $E_{1}: E_{2}: E_{3}$ is 10 : 10: 1 . The angel between incident probe beam and forward probe beam is $10^{\circ}$. The experimental setup was mounted on a vibration isolation table [14].

\section{Dye-Film Preparation}

The gelatin, supplied by Sigma Chemicals, is Type-A porcine skin with approximately 300 bloom. To produce dye doped gelatin film, 1 gram of gelatin is added to a $10 \mathrm{ml}$ solution of the dye and heated to about $5^{\circ} \mathrm{C}$ while stirring about $15 \mathrm{~min}$ to achieve a homogeneous solution. The solution was dispensed on the glass slide and agitated briefly to achieve an even delivery. Films were then allowed to be cooled. The thickness of films was about $40 \mu \mathrm{m}$ [8].

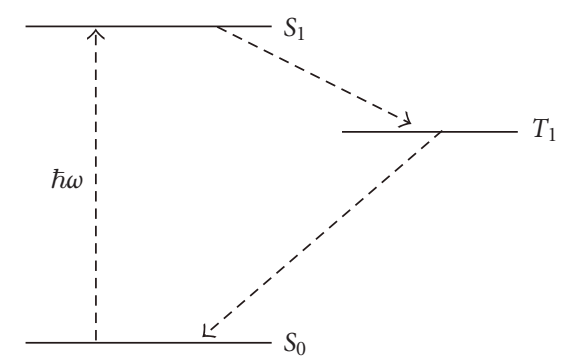

FIGURE 1: Three-level model for organic dyes.

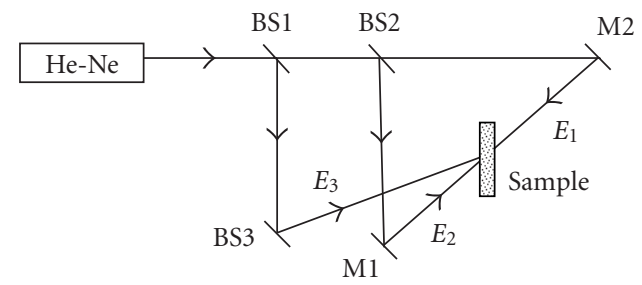

Figure 2: Experimental set-up for Observation of PC Wave.

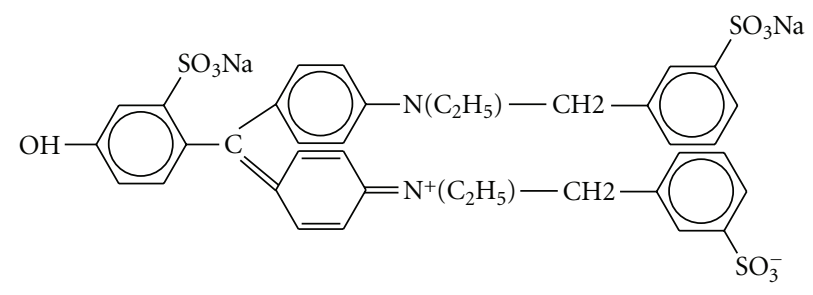

Figure 3: Molecular structure of acid blue 3.

\section{Material}

The organic dye Fast Green FCF (acid blue 3) belongs to the triphenylmethane groups. These groups are green, blue, or violet in colour [15]. The chemical structure and formula of this dye are shown in Figure 3. The UV-VIS absorption spectrum of Fast Green FCF dye exhibits the absorbance peak at $630 \mathrm{~nm}$ as shown in Figure 4. But the absorption peak is broad enough to allow us to work with $632 \mathrm{~nm}$ wavelength of He-Ne laser [13].

\section{Results and Discussion}

The PC signal measurements are taken by varying the parameters which influence the PC signal during the DFWM process. Figure 5 shows the PC signal versus the time for different dye concentration of the gelatin films. PC intensity rises linearly to a maximum and then starts decreasing. The phase grating formed is transient.

To get maximum reflectivities, it is necessary that there be a perfect overlap of the probe and the pump beams in the nonlinear medium. Figure 6 shows the PC reflectivities as a function of recording angle between the forward pump 


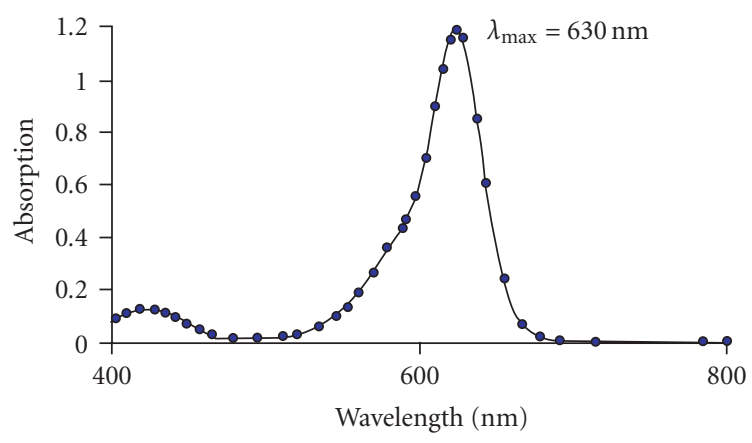

FIGURE 4: UV-VIS absorption spectrum of acid blue 3 dye in water.

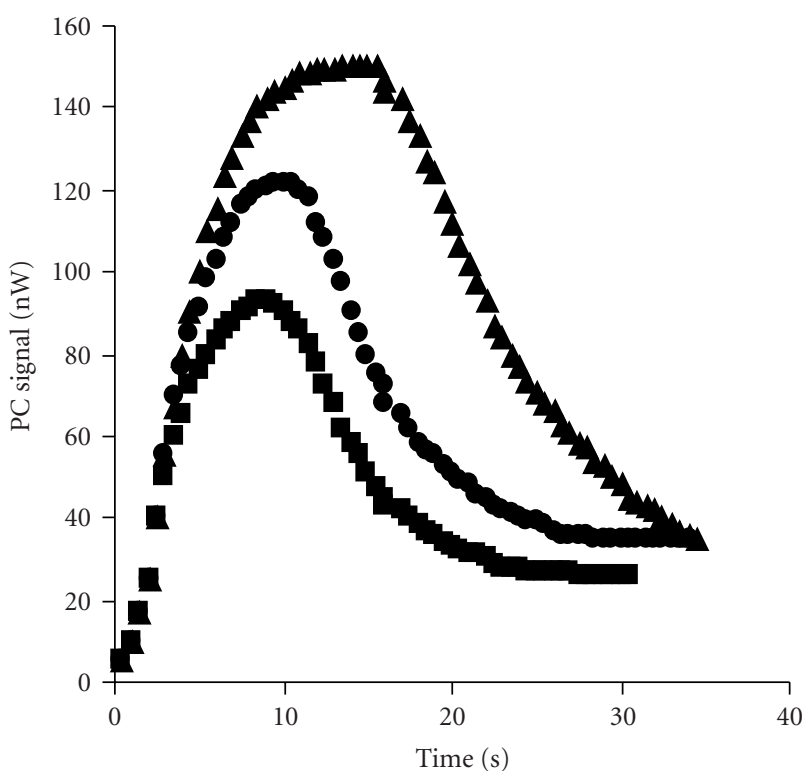
$\Delta 0.5 \mathrm{mM}$
- $1.5 \mathrm{mM}$
- $2 \mathrm{mM}$

FIGURE 5: PC signal versus time for different concentration.

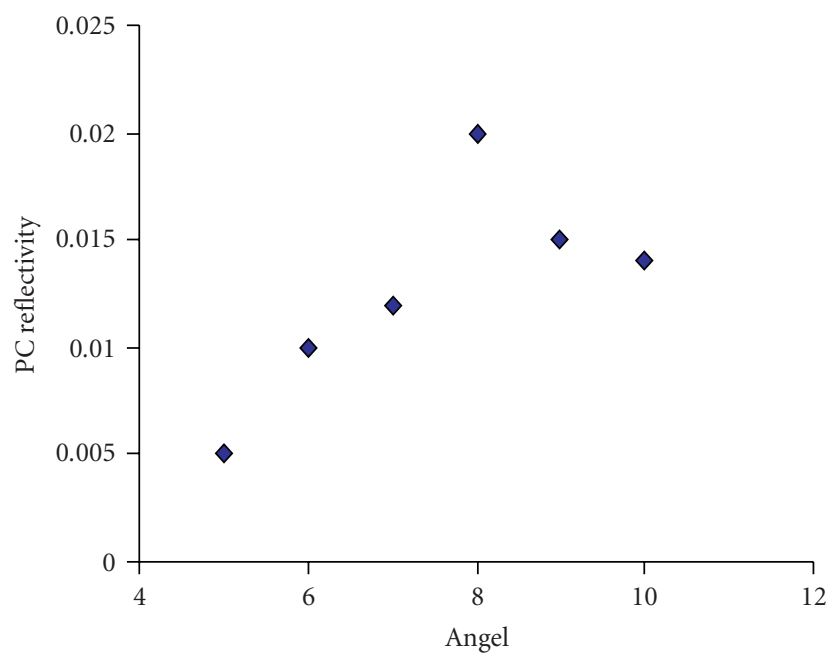

FIgURE 6: PC Reflectivity as function of angle between the probe beam and forward pump beam in acid blue 3 .

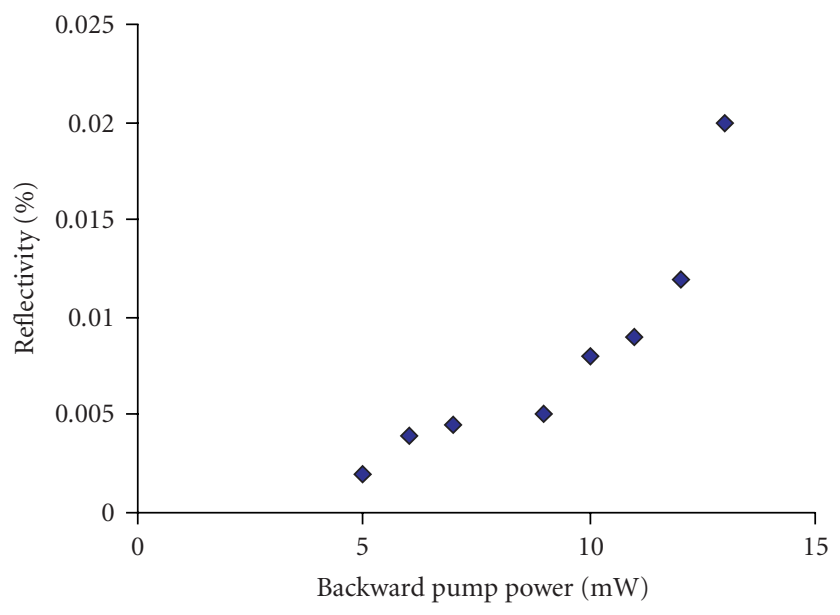

Figure 7: Dependence of PC reflectivity on backward pump power.

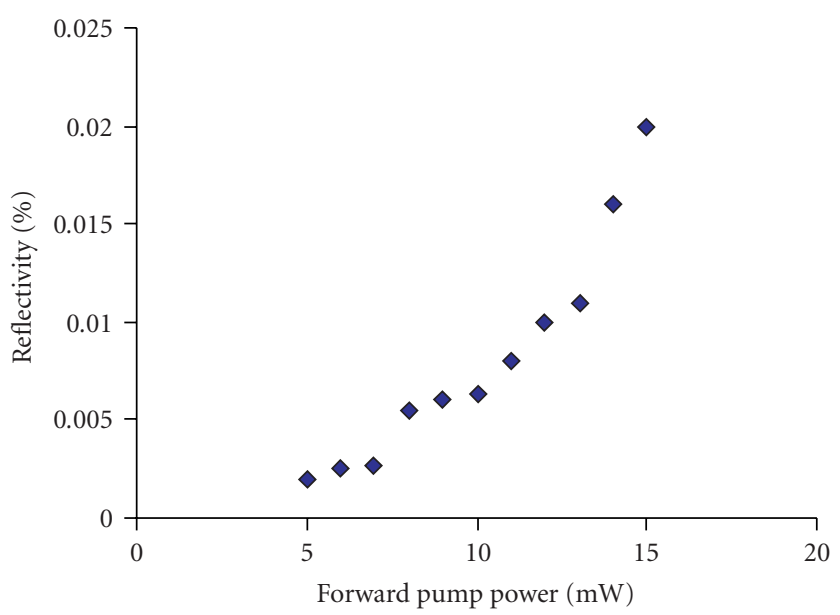

Figure 8: Dependence of PC reflectivity on forward pump power.

and probe beam. It seems from the figure that, as the angle between the probe beam and the forward pump beam increases, the PC reflectivity first increases and then decreases.

This may be because as the angle increases, the probe beam becomes elliptical and only a fraction of its area falls within the interaction region. Because of two-wave coupling, the maximum PC reflectivity is achieved when the angle is 8 degrees. We intend to study the effect of the backward pump beam power on the PC reflectivity by keeping the power of the forward pump and probe beams constant and varying the backward pump beam.

Figure 7 shows that higher backward powers are necessary to achieve higher reflectivity. The purpose is to study the dependence of the PC reflectivity on forward pump beam power by keeping the power of backward pump and probe beam constant and varying the forward pump power.

Figure 8 shows the variation of reflectivity for different power of forward pump beam. The PC reflectivity increases linearly with the power of forward pump beam. There are 


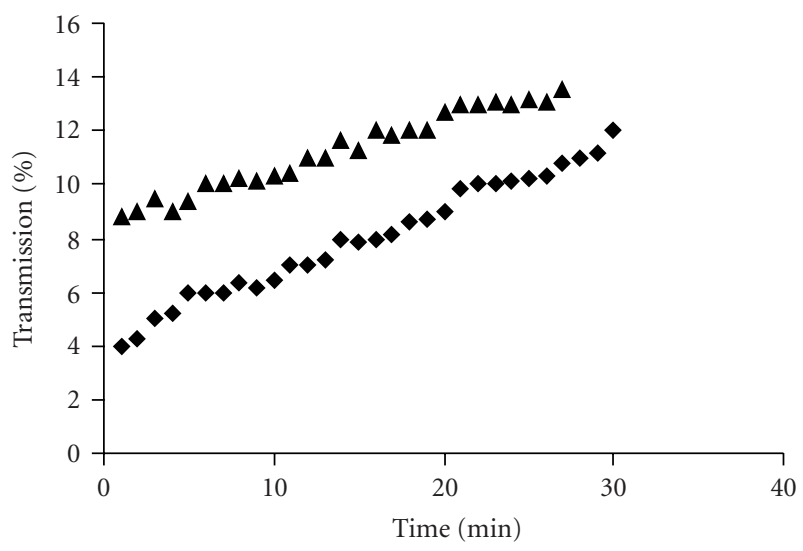

Figure 9: Transmission as a function of pump power in acid blue 3 .

two main processes which must be considered in the discussion of origin of OPC in gelatin films: (1) the formation of thermal grating and (2) photochemical bleaching.

The gelatin film illuminates with $632 \mathrm{~nm}$ radiation of variable intensity. The transmittance of the sample is measured simultaneously by using photodetector. If the effect observed in our experiments is of purely thermal nature, no bleaching will be observed. The results obtained for the sample are shown in Figure 9. It is clearly demonstrated that the transmission of sample increases. The experiment described above indicates that the photochemical nature of processes is mainly responsible for OPC in the sample under study.

\section{Conclusion}

In conclusion, we have observed low-intensity optical phase conjugation with acid blue 3 dye doped in gelatin films using $632 \mathrm{~nm}$ He-Ne wavelength. Though this dye gives good reflectivities, it suffers from low-damage threshold which may restrict its use for various applications. The effects of dye intensity backward, forward pump and interbeam angle between probe and forward pump beam on phase conjugation reflectivity are also studied. PC signal first increases and then decreases. PC reflectivity is increased by increasing the intensity of the backward and forward pump beam. The maximum PC reflectivity is achieved when the angle between probe and forward pump beam is 8 degrees. The gelatin film illuminates with radiation of variable intensity and transmittance of the sample is measured. A maximum phase-conjugate beam reflectivity of about $0.02 \%$ has been observed. Since the fast green FCF dye doped gelatin film is used at $632.8 \mathrm{~nm}$, it may be suitable for low power semiconductor laser in the red wavelength region.

\section{References}

[1] R. A. Fisher, Optical Phase Conjugation, Academic Press, New York, NY, USA, 1983.

[2] H. Tanaka, A. Horikoshi, H. Fujiwara, and K. Nakagawa, "Phase conjugation in saturable absorbing dye films by degenerate four-wave mixing and holographic processes using nanosecond pulse and CW lasers," Optical Review, vol. 9, no. 3, pp. 106-111, 2002.

[3] T. Geethakrishnan and P. K. Palanisamy, "Degenerate fourwave mixing experiments in Methyl green dye-doped gelatin film," Optik, vol. 117, no. 6, pp. 282-286, 2006.

[4] B. R. Reddy, P. Venkateswarlu, and M. C. George, "Laser induced gratings in a styryl due," Optics Communications, vol. 84, no. 5-6, pp. 334-338, 1991.

[5] M. A. Kramer, W. R. Tompkin, and R. W. Boyd, "Nonlinearoptical interactions in fluorescein-doped boric acid glass," Physical Review A, vol. 34, no. 3, pp. 2026-2031, 1986.

[6] Y. Silberberg and I. Bar-Joseph, "Transient effects in degenerate four-wave mixing in saturable absorbers," IEEE Journal of Quantum Electronics, vol. 17, no. 9, pp. 1967-1970, 1981.

[7] H. Eichler, G. Salje, and H. Stahl, "Thermal diffusion measurements using spatially periodic temperature distributions induced by laser light," Journal of Applied Physics, vol. 44, no. 12, pp. 5383-5388, 1973.

[8] R. Y. Choie, T. H. Barnes, W. J. Sandle, A. D. Woolhouse, and I. T. McKinnie, "Observation of a thermal phase-grating contribution to diffraction in erythrosin-doped gelatin films," Optics Communications, vol. 186, no. 1-3, pp. 43-50, 2000.

[9] R. L. Abrams and R. C. Lind, "Degenerate four-wave mixing in absorbing media," Optics Letters, vol. 2, no. 4, pp. 94-96, 1978.

[10] Y. Silberberg and I. Bar-Joseph, "Low power phase conjugation in thin films of saturable absorbers," Optics Communications, vol. 39, no. 4, pp. 265-268, 1981.

[11] R. W. Boyd, Nonlinear Optics, Academic Press, New York, NY, USA, 1992.

[12] A. Miniewicz, S. Bartkiewicz, J. Sworakowski, J. A. Giacometti, and M. M. Costa, "On optical phase conjugation in polystyrene films containing the azobenzene dye Disperse Red 1," Pure and Applied Optics, vol. 7, no. 4, pp. 709-721, 1998.

[13] T. Geethakrishnan and P. K. Palanisamy, "Optical phase conjugation and double-exposure phase-conjugate interferometry in dye-doped thin film using He-Ne laser," Applied Physics B, vol. 82, no. 2, pp. 169-172, 2006.

[14] P. R. Sreedhar and R. S. Sirohi, "Low power phase conjugation in phenosafranin dye films," Optics Communications, vol. 97, no. 1-2, pp. 83-87, 1993.

[15] K. Jamshidi-Ghaleh, S. Salmani, and M. H. Majles Ara, "Nonlinear responses and optical limiting behavior of fast green FCF dye under a low power CW He-Ne laser irradiation," Optics Communications, vol. 271, no. 2, pp. 551-554, 2007. 

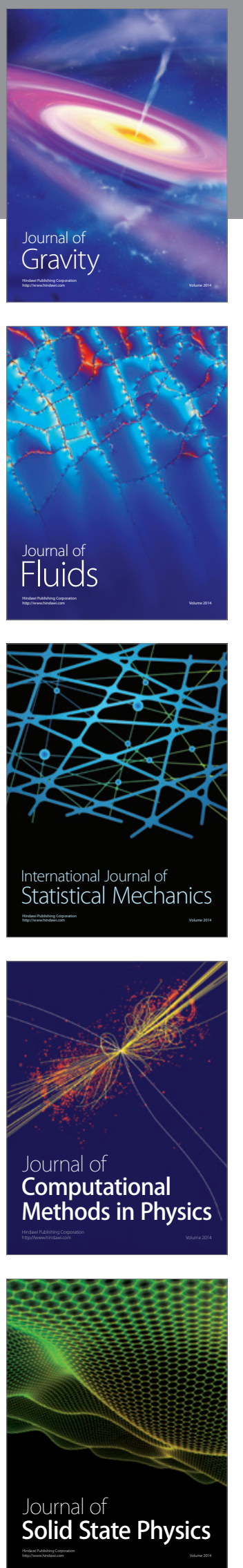

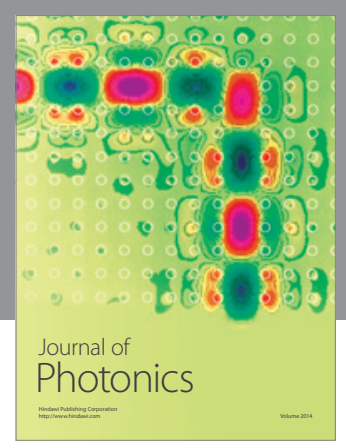

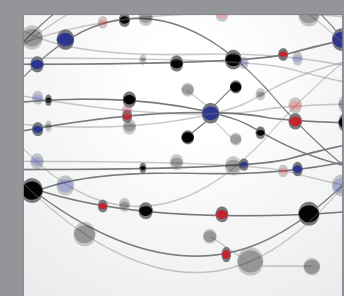

The Scientific World Journal
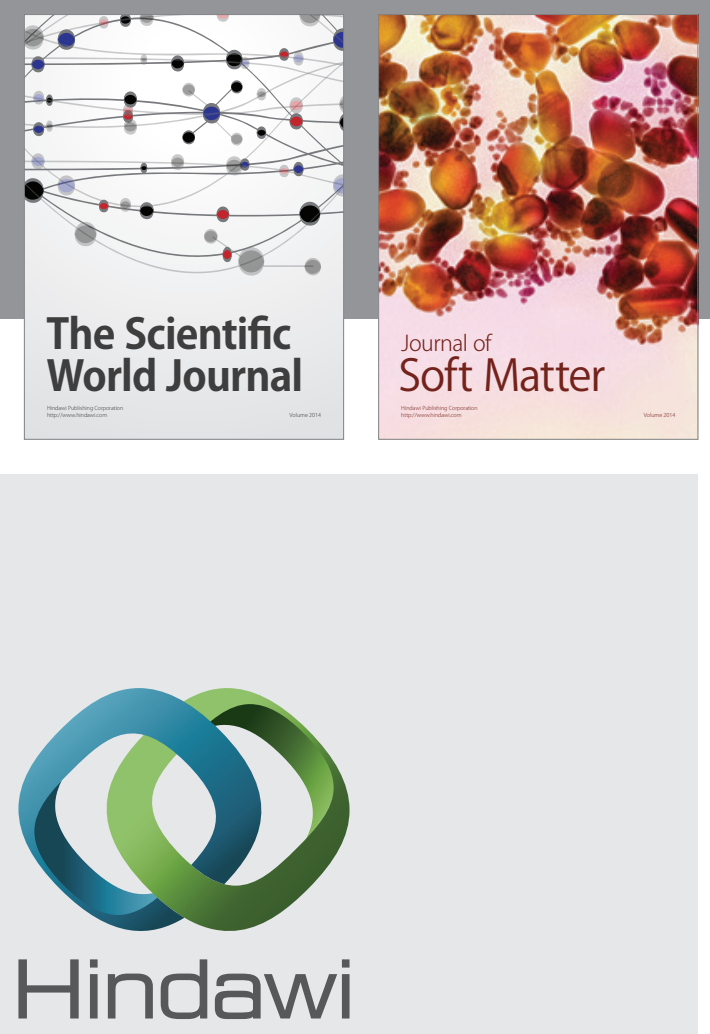

Submit your manuscripts at

http://www.hindawi.com
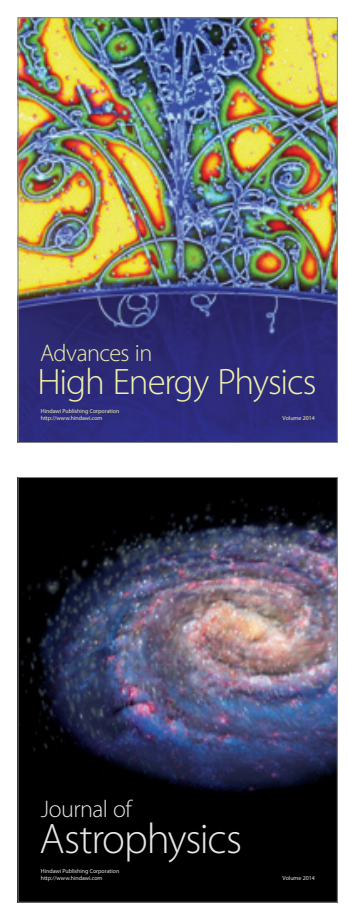
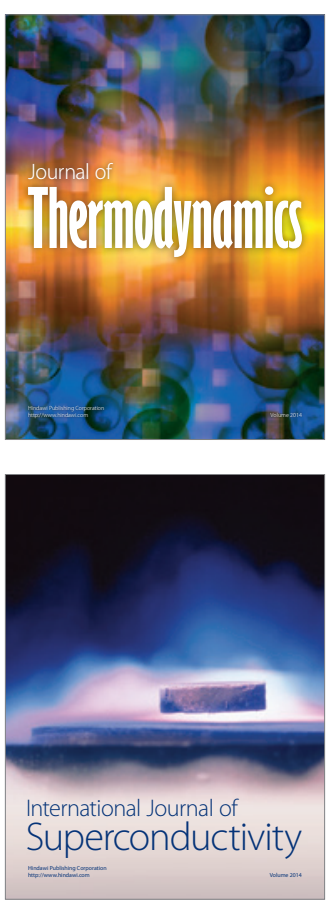
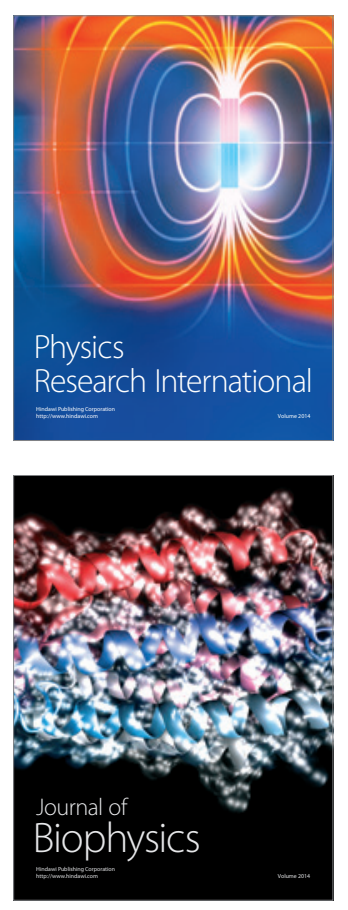
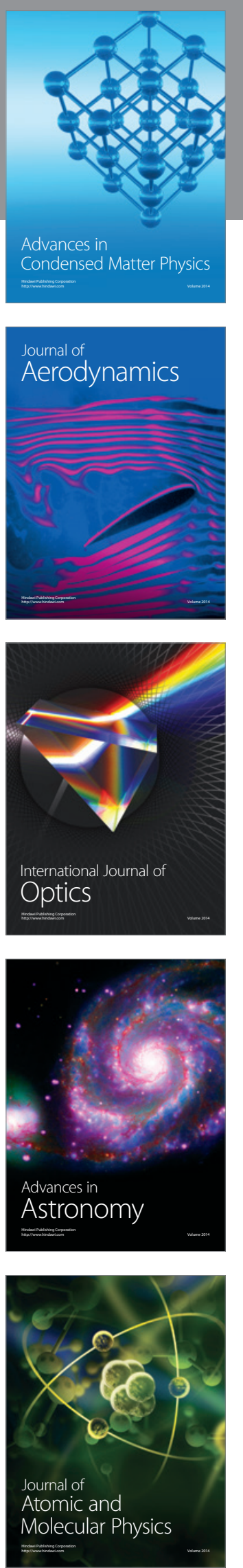